\title{
Laparoscopic low anterior resection using new articulating instruments
}

\author{
Chul Seung Lee ${ }^{1} \cdot$ Yoon Suk Lee ${ }^{1}$ (I)
}

Received: 7 May 2021 / Accepted: 13 June 2021 / Published online: 17 June 2021

(c) The Author(s) 2021

\begin{abstract}
Although total mesorectal excision (TME) has become the standard surgical treatment for mid and low rectal cancers, it has certain technical hurdles. TME in men can be technically challenging, even for experienced surgeons, as extensive incisions using subtle techniques and laparoscopic instruments are required to achieve a complex anastomosis. Obtaining an effective angle, traction, and countertraction is difficult with conventional straight-fixed laparoscopic instruments. To overcome these limitations, a surgical robot system was introduced $[1,2]$. This robotic system has the advantages of multi-joint mechanisms, ergonomics, and three-dimensional vision, but it is expensive. Several laparoscopic joint instruments have been introduced as alternatives to robotic systems [3]. In the attached video, we present laparoscopic low anterior resection performed by simultaneously using two laparoscopic articulating instruments.

We show the use of two articulating laparoscopic instruments (ArtiSential ${ }^{\circledR}$, LIVSMED, Inc., Republic of Korea) which were registered as class I medical devices with the Food and Drug Administration in 2019 and have been available since November 2019 in Korea. The patient was a 62-year-old man with moderately differentiated adenocarcinoma of the mid rectum, clinical stage $\mathrm{T} 3 \mathrm{~N} 1$ and no evidence of distant metastasis. We performed laparoscopic low anterior resection with TME. The patient was placed in a modified lithotomy position, and five trocars were used: one 10-mm umbilical trocar for the laparoscope port, one 12-mm trocar on the left lower quadrant, one 8-mm trocar in the upper quadrant, and two additional 5-mm trocars used as described in Fig. 1. During the procedures, the surgeon used the two ArtiSential® instruments through the right
\end{abstract}

Yoon Suk Lee

yslee@ catholic.ac.kr

1 Division of Colorectal Surgery, Department of Surgery, Seoul St. Mary's Hospital, College of Medicine, The Catholic University of Korea, 222, Banpo-daero, Seocho-gu, Seoul 06591, Republic of Korea lower and upper quadrant ports (Fig. 2). Using the 5-trocar approach, the inferior mesenteric vessels were ligated after identifying the left ureter, followed by retromesenteric dissection using a medial-to-lateral route. The splenic flexure was then mobilized, followed by laparoscopic TME dissection with preservation of the hypogastric plexus and nerves. For tumors located in the distal rectum $(6 \mathrm{~cm}$ from the anal verge), a complete TME was performed laparoscopically after splenic flexure mobilization. The rectum was transected using an endoscopic stapler. The umbilical port was extended, and the specimen was extracted and resected with an adequate oncological proximal margin. Intracorporeal end-to-end anastomosis was performed after checking the perfusion status using indocyanine green.

There were no intra- or postoperative complications. The final pathological diagnosis was pT2N0M0. Thirteen regional lymph nodes were harvested, and no metastatic regional lymph nodes were identified.

Conventional straight-fixed laparoscopic instruments have the disadvantages of reduced dexterity, limited freedom of movement, and uncomfortable ergonomics [4]. It is sometimes challenging for surgeons to obtain an effective angle, traction, or countertraction during laparoscopic surgery. Although the surgical robot system solves these problems, there is an issue of cost-effectiveness [1,2]. A new laparoscopic joint instrument (ArtiSential®, LIVSMED, Inc., South Korea) helps surgeons easily obtain effective traction and countertraction through intuitive movements. The instrument is synchronized with the user's hand and can be moved at a $360^{\circ}$ angle, allowing for more versatile surgical procedures than a straight-fixed laparoscopic instrument [3].

We present a standardized procedure for laparoscopic low anterior resection with TME using two new laparoscopic articulating instruments. One of the most important technical advantages of TME is the effective traction and countertraction it provides in the narrow pelvis. Unfortunately, in the presence of bulky tumors, a narrow male pelvis, or obesity, a surgical approach with conventional laparoscopic 
Fig. 1 Placement of trocars

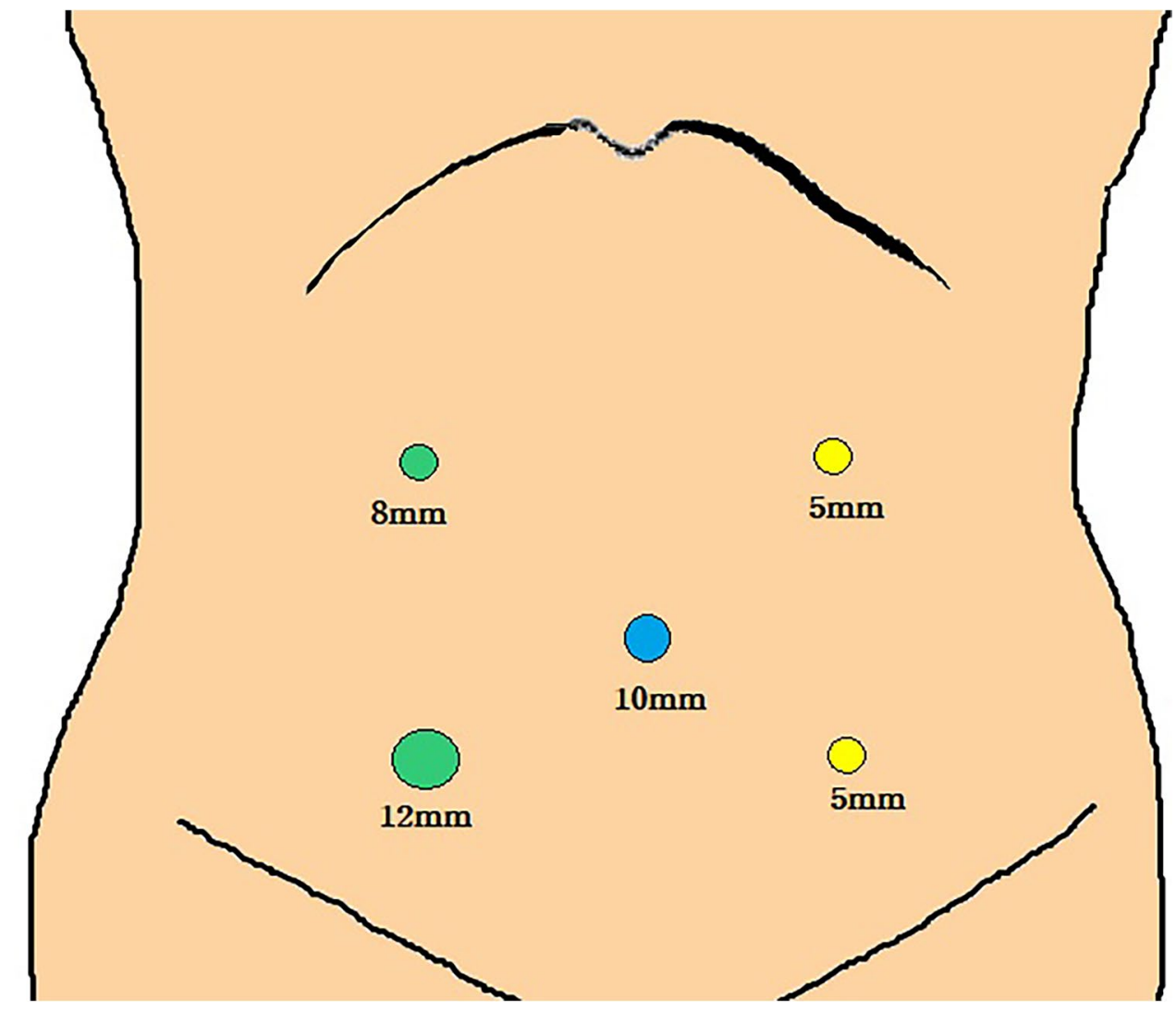

instruments is technically more challenging [5]. The use of two articulating laparoscopic instruments could be particularly helpful for exposing surgical planes and skeletonizing the primary feeding vessels.

This is the first video presenting the simultaneous clinical application of two newly released articulating laparoscopic instruments (ArtiSential®, LIVSMED, Inc., Republic of
Korea). We show that laparoscopic low anterior resection using two articulating laparoscopic instruments is safe and technically feasible. These instruments are less expensive than a robot system, but like robot surgery, ergonomic and intuitive surgery is possible with multiple joints. Future comparative studies with robotic surgery are needed to demonstrate its benefits for clinical applications. 

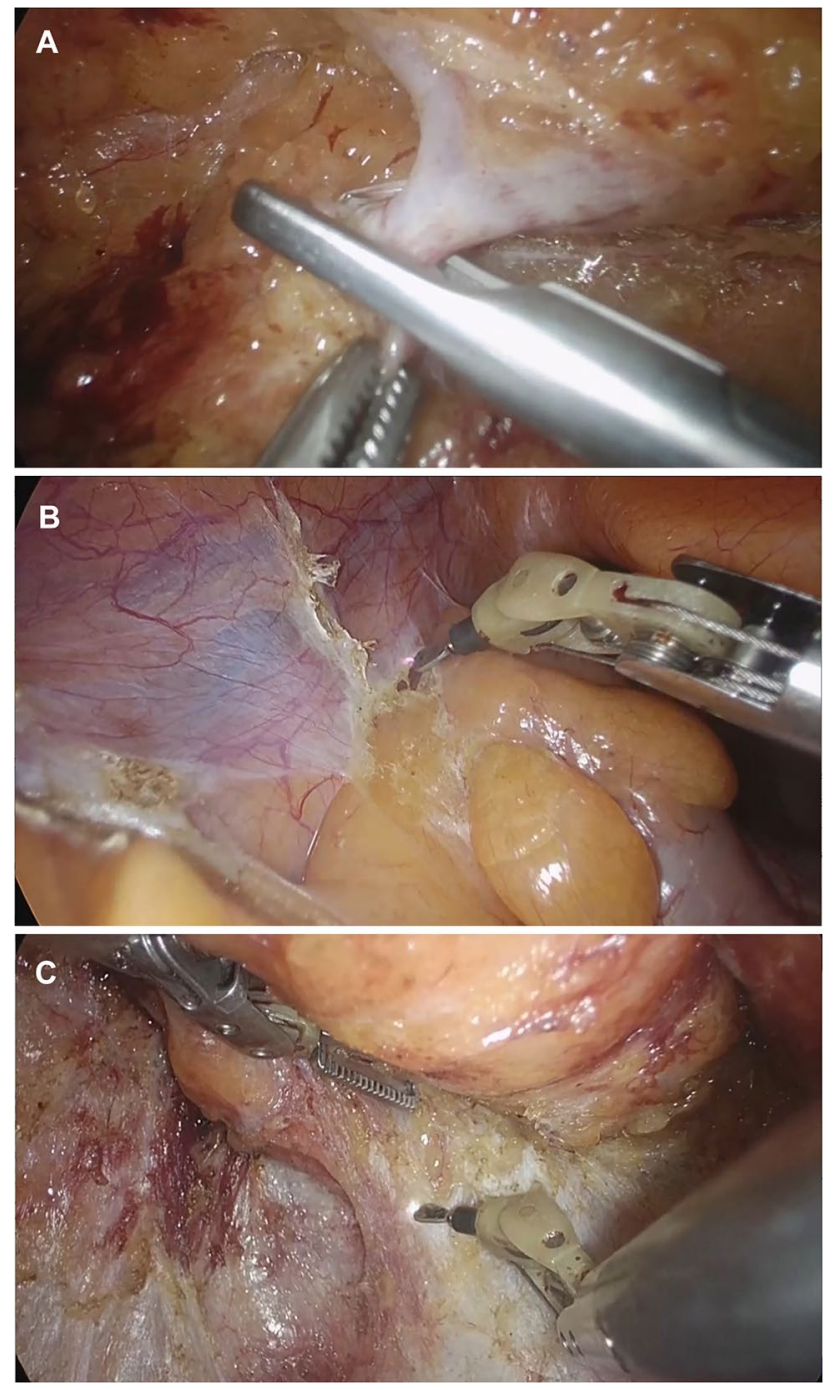

Fig. 2 Mesenteric lymphadenectomy and total mesorectal excision. A High ligation of the inferior mesenteric artery; $\mathbf{B}$ lateral dissection; $\mathbf{C}$ total mesorectal excision

Supplementary Information The online version contains supplementary material available at https://doi.org/10.1007/s10151-021-02486-9.

Funding This study was not funded.

\section{Declarations}

Conflict of interest The authors declare that they have no conflict of interest.
Ethical approval This study was approved by the Seoul ST. Mary's Hospital Research Ethics Board.

Informed consent Informed consent was obtained from the patient (No. KC21ZASI0290).

Open Access This article is licensed under a Creative Commons Attribution 4.0 International License, which permits use, sharing, adaptation, distribution and reproduction in any medium or format, as long as you give appropriate credit to the original author(s) and the source, provide a link to the Creative Commons licence, and indicate if changes were made. The images or other third party material in this article are included in the article's Creative Commons licence, unless indicated otherwise in a credit line to the material. If material is not included in the article's Creative Commons licence and your intended use is not permitted by statutory regulation or exceeds the permitted use, you will need to obtain permission directly from the copyright holder. To view a copy of this licence, visit http://creativecommons.org/licenses/by/4.0/.

\section{References}

1. Park SY, Choi GS, Park JS, Kim HJ, Choi WH, Ryuk JP (2012) Robot-assisted right colectomy with lymphadenectomy and intracorporeal anastomosis for colon cancer: technical considerations. Surg Laparosc Endosc Percutan Tech 22:e271-276. https://doi.org/ 10.1097/SLE.0b013e31826581bd

2. Baek SJ, Kim SH, Cho JS, Shin JW, Kim J (2012) Robotic versus conventional laparoscopic surgery for rectal cancer: a cost analysis from a single institute in Korea. World J Surg 36:2722-2729. https://doi.org/10.1007/s00268-012-1728-4

3. Jin HY, Lee CS, Lee YS (2021) Laparoscopic extended right hemicolectomy with D3 lymph node dissection using a new articulating instrument. Tech Coloproctol 25:235-237. https:// doi.org/10.1007/s10151-020-02345-z

4. Moore LJ, Wilson MR, McGrath JS, Waine E, Masters RS, Vine SJ (2015) Surgeons' display reduced mental effort and workload while performing robotically assisted surgical tasks, when compared to conventional laparoscopy. Surg Endosc 29:2553-2560. https://doi.org/10.1007/s00464-014-3967-y

5. Garlipp B, Ptok H, Schmidt U, Stübs P, Scheidbach H, Meyer F, Gastinger I, Lippert H (2012) Factors influencing the quality of total mesorectal excision. Br J Surg 99:714-720. https://doi.org/ 10.1002/bjs.8692

Publisher's Note Springer Nature remains neutral with regard to jurisdictional claims in published maps and institutional affiliations. 